\title{
Investigations in the possibility of early detection of colorectal cancer by gas chromatography/triple-quadrupole mass spectrometry
}

\author{
Shin Nishiumi ${ }^{1, *}$, Takashi Kobayashi1, ${ }^{1}$, Shuichi Kawana ${ }^{2}$, Yumi Unno ${ }^{2}$, Takero \\ Sakai $^{2}$, Koji Okamoto ${ }^{3}$, Yasuhide Yamada ${ }^{4}$, Kazuki Sudo ${ }^{4}$, Taiki Yamaji ${ }^{5}$, Yutaka \\ Saito6, Yukihide Kanemitsu7, Natsuko Tsuda Okita4, Hiroshi Saito8, Shoichiro \\ Tsugane ${ }^{9}$, Takeshi Azuma ${ }^{1}$, Noriyuki Ojima², Masaru Yoshida ${ }^{1,10,11}$ \\ ${ }^{1}$ Division of Gastroenterology, Department of Internal Medicine, Kobe University Graduate School of Medicine, Chuo-ku, \\ Kobe, Hyogo 650-0017, Japan \\ ${ }^{2}$ Analytical and Measuring Instruments Division, Shimadzu Corporation, Nakagyo-ku, Kyoto 604-8511, Japan \\ ${ }^{3}$ Division of Cancer Differentiation, National Cancer Center Research Institute, Tokyo 104-0045, Japan \\ ${ }^{4}$ Department of Gastrointestinal Medical Oncology, National Cancer Center Hospital, Chuo-ku, Tokyo 104-0045, Japan \\ ${ }^{5}$ Division of Epidemiology, Center for Public Health Sciences, National Cancer Center, Chuo-ku, Tokyo 104-0045, Japan \\ ${ }^{6}$ Endoscopy Division, National Cancer Center Hospital, Chuo-ku, Tokyo 104-0045, Japan \\ ${ }^{7}$ Department of Colorectal Surgery, National Cancer Center Hospital, Chuo-ku, Tokyo 104-0045, Japan \\ ${ }^{8}$ Division of Screening Assessment and Management, Center for Public Health Sciences, National Cancer Center, Chuo-ku, \\ Tokyo 104-0045, Japan \\ ${ }^{9}$ Center for Public Health Sciences, National Cancer Center, Chuo-ku, Tokyo 104-0045, Japan \\ ${ }^{10}$ Division of Metabolomics Research, Department of Internal Related, Kobe University Graduate School of Medicine, Chuo- \\ ku, Kobe, Hyogo 650-0017, Japan \\ ${ }^{11}$ AMED-CREST, AMED, Chuo-ku, Kobe, Hyogo 650-0017, Japan \\ *These authors have contributed equally to this work \\ Correspondence to: Masaru Yoshida, email: myoshida@med.kobe-u.ac.jp \\ Keywords: metabolomics, colorectal cancer, biomarker, gas chromatography/triple-quadrupole mass spectrometry, metabolite \\ Received: November 14, $2016 \quad$ Accepted: January 09, $2017 \quad$ Published: February 04, 2017
}

\section{ABSTRACT}

In developed countries, the number of patients with colorectal cancer has been increasing, and colorectal cancer is one of the most common causes of cancer death. To improve the quality of life of colorectal cancer patients, it is necessary to establish novel screening methods that would allow early detection of colorectal cancer. We performed metabolome analysis of a plasma sample set from 282 stage 0/I/II colorectal cancer patients and 291 healthy volunteers using gas chromatography/ triple-quadrupole mass spectrometry in an attempt to identify metabolite biomarkers of stage $0 / \mathrm{I} / \mathrm{II}$ colorectal cancer. The colorectal cancer patients included patients with stage $0(\mathrm{~N}=79), I(\mathrm{~N}=80)$, and II $(\mathrm{N}=123)$ in whom invasion and metastasis were absent. Our analytical system detected 64 metabolites in the plasma samples, and the levels of 29 metabolites differed significantly (Bonferroni-corrected $p=0.000781$ ) between the patients and healthy volunteers. Based on these results, a multiple logistic regression analysis of various metabolite biomarkers was carried out, and a stage $0 / \mathrm{I} / \mathrm{II}$ colorectal cancer prediction model was established. The area under the curve, sensitivity, and specificity values of this model for detecting stage $0 / \mathrm{I} /$ II colorectal cancer were $0.996,99.3 \%$, and $93.8 \%$, respectively. The model's sensitivity and specificity values for each disease stage were $>90 \%$, and surprisingly, its sensitivity for stage 0 , specificity for stage 0 , and sensitivity for stage II disease 


\section{were all $100 \%$. Our predictive model can aid early detection of colorectal cancer and has potential as a novel screening test for cases of colorectal cancer that do not involve lymph node or distant metastasis.}

\section{INTRODUCTION}

In developed countries, colorectal cancer is one of the most common causes of cancer death [1], and it is treated using a combination of colonoscopy, surgery, chemotherapy, and radiotherapy. When colorectal cancer is discovered early, its 5-year relative survival rate is very high, but advanced colorectal cancer reduces the quality of life (QOL) of patients. Therefore, novel methods that would allow the early detection and diagnosis of colorectal cancer are desired in the medical field. The fecal occult blood test (FOBT) and blood tests for tumor markers are commonly used as screening methods for diagnosing colorectal cancer. The FOBT is a non-invasive and inexpensive method, but has low sensitivity and specificity for cases of colorectal cancer that do not involve lymph node or distant metastasis. As for blood tests for tumor markers, carcinoembryonic antigen (CEA) and carbohydrate antigen 19-9 (CA19-9) can be used to test for colorectal cancer, but such blood tests also have low sensitivity so they are not appropriate for detecting colorectal cancer early. Colonoscopy is a more accurate and reliable method for detecting colorectal cancer early, but it cannot be used for screening because it is more invasive and expensive than blood testing. Recently, computed tomographic colonography, which is less invasive than colonoscopy, has been used to detect colorectal cancer; however, special X-ray equipment is required to obtain detailed scans, which is expensive. Combinations of conventional screening methods are also used to detect colorectal cancer, but such combined approaches only detect about $40 \%$ of colorectal cancer cases [2].Therefore, it is necessary to establish the novel screening methods for early detection of colorectal cancer with high sensitivity, high specificity, noninvasive and easy procedure.

Biomarker research into colorectal cancer is being carried out using a variety of approaches including omicsbased methods, such as genomics and proteomics, and many researchers are searching for novel biomarkers that would allow the early detection of the disease and could also be used for predicting therapeutic efficacy, recurrence, and prognosis, etc. [3]. In our previous study, serum metabolomics or metabolome analysis, which is the comprehensive study of low molecular weight metabolites, was employed to find novel metabolite biomarkers of colorectal cancer, and then a colorectal cancer prediction model based on four metabolites was established using multiple logistic regression analysis [4]. The metabolome mainly represents the endpoint of the omics cascade, and it is also the closest point in the cascade to the phenotype. The genome, which is located in the upstream part of the omics cascade and includes numerous genes, is generally not affected by exogenous factors, such as environmental and dietary factors. Even if a certain gene contains a mutation, it might not affect the rest of the body due to the effects of homeostatic functions. In addition to variations in DNA, mRNA, and protein expression, the metabolome is affected by the enzymatic activities of various proteins, and alterations in the levels of metabolites can also be caused by exogenous factors. Therefore, the metabolome could be considered to be a summary of the other upstream omics profiles, and metabolome analysis might be able to detect subtle changes in metabolic pathways and deviations from homeostasis before phenotypic changes occur [5,6]. Thus, metabolomics could contribute greatly to biomarker research $[7,8]$. Our previous study [4], in which a colorectal cancer prediction model was established, included colorectal cancer patients with stage 0 to IV disease, and the colorectal cancer prediction model demonstrated sensitivity and specificity values of about $80 \%$. However, the number of stage $0 / \mathrm{I} / \mathrm{II}$ colorectal cancer patients was relatively small, so it is necessary to carry out a further trial involving a larger number of stage $0 / \mathrm{I} / \mathrm{II}$ colorectal cancer patients in order to establish a screening procedure that could contribute to the early detection of colorectal cancer and improve the QOL of colorectal cancer patients.

In this study, we employed gas chromatography/ triple-quadrupole mass spectrometry (GC/QqQMS), which is generally used for multiple reaction monitoring (MRM) analysis. QqQMS-based MRM analysis can be used to easily distinguish single metabolite-derived peaks from co-eluted peaks and background noise, and it exhibits high sensitivity and a wide dynamic range compared with single-QMS [9], although gas chromatography/ single-quadrupole mass spectrometry (GC/QMS) is very useful for comprehensive metabolite analysis [10]. In our previous study [4], GC/QMS was used to discover biomarker candidates for colorectal cancer. An automatic derivatization machine was also used because intersample differences in the time between derivatization and measurement can affect the results [11, 12]. By using this automatic derivatization machine, our GC/QqQMS analysis system is able to obtain more accurate metabolite data. The aim of the present study is to find metabolite biomarker candidates that would allow the detection of cases of colorectal cancer that do not involve lymph node or distant metastasis. To do this, human plasma samples that were collected from stage 0 to stage II colorectal cancer patients and corresponding healthy volunteers were analyzed using our GC/QqQMS analysis system. Then, a multiple logistic regression model for detecting cases of colorectal cancer without any lymph node or distant metastasis was established on the basis of the metabolite data. 


\section{RESULTS}

Our GC/QqQMS-based metabolite analysis system detected 64 metabolites in the subjects' plasma. The sample set examined in this study was obtained from 282 colorectal cancer patients and 291 healthy volunteers, and the colorectal cancer patients included clinical stage $0(\mathrm{~N}=79), \mathrm{I}(\mathrm{N}=80)$, and II $(\mathrm{N}=123)$ patients who were free from invasion and metastasis. Regarding the characteristics of the colorectal cancer patients and healthy volunteers included in this study (Table 1), there were no significant differences in age or body mass index (BMI) between the colorectal cancer patients and healthy volunteers. The male-to-female ratio of the two groups also did not differ significantly. Significant differences in the frequencies of current smokers, former smokers, and people who had never smoked were observed between the colorectal cancer patients and healthy volunteers, whereas the significance of the inter-group differences in the frequencies of current alcohol drinkers, former alcohol drinkers, and people who had never drank alcohol could not be confirmed. The numbers of people who were and were not taking medication differed significantly between the colorectal cancer patients and healthy volunteers. As for the blood test results, the blood levels of total cholesterol, triglycerides, glucose, total bilirubin, aspartate transaminase (AST), C-reactive protein (CRP), CEA, and CA19-9 differed significantly between the colorectal cancer patients and healthy volunteers (Supplementary Table 1).

The plasma metabolite levels of the colorectal cancer patients and healthy volunteers were compared using Wilcoxon's rank sum test (Supplementary Table 2). As a result, the plasma levels of 41 of the 64 metabolites were shown to differ significantly $(p<0.05)$ between the colorectal cancer patients and healthy volunteers. After the application of Bonferroni's correction ( $\mathrm{p}=0.000781)$, the levels of 29 metabolites continued to exhibit significant differences. Next, a simple linear regression analysis of the 29 metabolites that demonstrated Bonferroni-corrected significant differences was performed, and their area under the curve (AUC), sensitivity, and specificity values were evaluated (Table 2). Pyruvic acid-meto-trimethylsilyl (TMS), glycolic acid-2TMS, lactic acid-2TMS(/SI), and fumaric acid-2TMS(/SI) displayed AUC values of $>0.8$, and both the sensitivity and specificity of pyruvic acidmeto-TMS and lactic acid-2TMS(/SI) were $>80 \%$.

Based on these results, multiple logistic regression analysis involving various metabolite biomarkers was performed. The 29 metabolites that exhibited Bonferronicorrected significant inter-group differences were subjected to a stepwise variable selection method, and 8 metabolites (pyruvic acid-meto-TMS, glycolic acid2TMS, tryptophan-3TMS(/SI), palmitoleic acid-TMS, fumaric acid-2TMS(/SI), ornithine-4TMS(/SI), lysine4TMS, and 3-hydroxyisovaleric acid-2TMS) were selected as variables for the multiple logistic regression model. Then, a multiple logistic regression model composed of these 8 selected metabolites was established, which resulted in the following predictive model (Figure 1):

$\mathrm{p}=1 /\left[1+\exp ^{-\{-8.99+19.38 \times 1+82.33 \times 2-6.86 \times 3-67.48 \times 4+296.32 \times 5+9.69 \times 6-}\right.$ 5.76X7-24.99X8\};

The AUC, sensitivity, and specificity values of this model were $0.996,99.3 \%$, and $93.8 \%$, respectively (Table 3). On the other hand, the sensitivity of CEA and CA19-9 were both $<20 \%$, although the specificity of CEA and CA19-9 were both $>95 \%$ (Table 3). The sensitivity/ specificity of the developed model for each clinical stage (0/I/II) were also evaluated (Table 3$)$. As a result, it was found that the model exhibited sensitivity and specificity values of $>90 \%$ for each stage of the disease, and surprisingly its sensitivity for stage 0 disease, specificity for stage 0 disease, and sensitivity for stage II disease were all $100 \%$. On the contrary, the sensitivity of CEA was $3.8 \%$ for stage 0 disease, $15.0 \%$ for stage I disease, and $29.3 \%$ for stage II disease, and the sensitivity of CA19-9 was $6.4 \%$ for stage 0 disease, $6.3 \%$ for stage I disease, and $13.1 \%$ for stage II disease. CEA and CA19-9 exhibited specificity values of $>90 \%$ for each stage of the disease.

Finally, the AUC of the predictive model was compared with that of pyruvic acid-meto-TMS (Supplementary Figure 1) because the AUC of pyruvic acid-meto-TMS was especially high in the simple linear regression analysis. As a result, the AUC of the predictive model was shown to be significantly superior to that of pyruvic acid-meto-TMS $(\mathrm{p}<0.0001)$.

\section{DISCUSSION}

In this study, we investigated whether the alterations in plasma metabolite levels are available for early detection of colorectal cancer. An analytical system composed of an automatic derivatization machine and GC/ QqQMS was used for this investigation. The automatic derivatization machine helped to eliminate the influence of inter-sample differences in the time between derivatization and measurement on plasma metabolite levels. In addition, GC/QqQMS makes it possible to easily distinguish the peak for a particular metabolite from co-eluted peaks and background noise, resulting in higher quality evaluations. The aim of this study is to find metabolite biomarkers and/ or multiple metabolite-based multiple logistic regression models that would allow the discovery and diagnosis of cases of colorectal cancer that do not involve lymph node or distant metastasis. Therefore, the sample set was composed of colorectal cancer patients with stage 0 , I, or II disease and corresponding healthy volunteers.

Some previous metabolomics studies have attempted to discover biomarkers that might aid the early detection of colorectal cancer. Ritchie et al. performed non-targeted and targeted serum metabolite profiling using training sets composed of three and two independent 
Table 1: Characteristics of the colorectal cancer patients and healthy volunteers

\begin{tabular}{|c|c|c|c|c|}
\hline & & CRC & HV & \multirow{2}{*}{ p-value } \\
\hline & $\mathbf{N}$ & 282 & 291 & \\
\hline \multirow{3}{*}{ Age (y.o.) } & Median & $68(40-93)$ & $68(41-88)$ & \multirow{3}{*}{0.825} \\
\hline & Mean & 67.0 & 66.8 & \\
\hline & S.D. & 9.02 & 7.94 & \\
\hline \multirow{2}{*}{ Sex } & Male & 170 & 178 & \multirow{2}{*}{0.828} \\
\hline & Female & 112 & 113 & \\
\hline \multirow{2}{*}{ BMI $\left(\mathrm{kg} / \mathrm{m}^{2}\right)$} & Mean & 22.9 & 22.8 & \multirow{2}{*}{0.6119} \\
\hline & S.D. & 3.62 & 2.82 & \\
\hline \multirow{3}{*}{ Smoking habits } & Current & 52 & 24 & \multirow{3}{*}{0.0006} \\
\hline & Former & 116 & 118 & \\
\hline & Never & 114 & 149 & \\
\hline \multirow{4}{*}{ Alcohol consumption } & Current & 174 & 198 & \multirow{4}{*}{0.1132} \\
\hline & Former & 26 & 18 & \\
\hline & Never & 79 & 75 & \\
\hline & N.R. & 3 & 0 & \\
\hline \multirow{2}{*}{ Medication } & Yes & 160 & 202 & \multirow{2}{*}{0.0017} \\
\hline & No & 122 & 89 & \\
\hline \multirow{6}{*}{ Tumor (T) } & Tis & 79 & & \\
\hline & $\mathrm{T} 1$ & 53 & & \\
\hline & $\mathrm{T} 2$ & 27 & & \\
\hline & $\mathrm{T} 3$ & 119 & & \\
\hline & $\mathrm{T} 4 \mathrm{a}$ & 2 & & \\
\hline & $\mathrm{T} 4 \mathrm{~b}$ & 2 & & \\
\hline \multirow{2}{*}{ Lymph node (N) } & N0 & 282 & & \\
\hline & $\mathrm{N} 1 / 2$ & 0 & & \\
\hline \multirow{2}{*}{ Metastasis (M) } & M0 & 282 & & \\
\hline & M1 & 0 & & \\
\hline \multirow{3}{*}{ Stage } & 0 & 79 & & \\
\hline & I & 80 & & \\
\hline & II & 123 & & \\
\hline
\end{tabular}

(Continued) 


\begin{tabular}{lcccc}
\hline & & CRC & HV & p-value \\
\cline { 2 - 3 } Site & N & $\mathbf{2 8 2}$ & 291 & \\
\hline C & 33 & \\
A & 39 & \\
T & 23 & \\
D & 11 & \\
S & 73 & \\
R & 101 & \\
P & 2 & \\
& tub1 & & \\
Histology & tub2 & 175 & \\
por1 & 91 & \\
por2 & 2 & \\
pap & 1 & \\
& muc & 3 & \\
\hline
\end{tabular}

The p-values for age and BMI were calculated using Welch's t-test. The p-values for sex, smoking habits, alcohol consumption, medication usage, and cancer stage were calculated using Pearson's chi-squared test. CRC: colorectal cancer patients; HV: healthy volunteers; y.o.: years old; BMI: body mass index; S.D.: standard deviation; N.R.: no response; C: cecum; A: ascending colon; T: transverse colon; D: descending colon; S: sigmoid colon; R: rectum; P: sacral promontory.

cohorts, respectively, and 28-36 carbon-containing hydroxylated polyunsaturated ultra-long-chain fatty-acids were identified as biomarkers of colorectal cancer [13]. In this study by Ritchie et al., 13 to 34 stage 0/I/II colorectal cancer patients were included in each cohort, and 2836 carbon-containing hydroxylated polyunsaturated ultra-long-chain fatty acids were shown to be useful for discovering stage 0/I/II colorectal cancer patients. In a study by Miyagi et al., two-class linear discrimination analysis based on plasma amino acid profiling showed AUC values of $0.903,0.859$, and 0.829 for detecting stage $0(n=8), I(n=63)$, and II $(n=48)$ colorectal cancer, respectively [14]. A study by Fang et al., which included 26 stage I/II colorectal cancer patients, found that the serum levels of some lipid species differed significantly between stage I/II colorectal cancer patients and healthy volunteers [15]. Proton nuclear magnetic resonance spectroscopy ( ${ }^{1} \mathrm{HNMR}$ )-based fecal metabolomic fingerprinting was reported to be useful for distinguishing stage I/II colorectal cancer patients from healthy volunteers in a study involving 20 stage I/II colorectal cancer patients [16]. In a study by Uchiyama et al. based on capillary electrophoresis/mass spectrometry [17], in which 14 stage I colorectal cancer patients and 14 stage II colorectal cancer patients were included in the sample set, the serum level of benzoic acid showed sensitivity, specificity, and AUC values of 1.0, 0.98, and 0.98, respectively, for stage I colorectal cancer, and $0.93,0.90$, and 0.95 , respectively, for stage II colorectal cancer. Therefore, previous studies have identified promising metabolite biomarkers of cases of colorectal cancer that do not involve lymph node or distant metastasis, but many of these studies included small numbers of colorectal cancer patients that were free from lymph node and distant metastasis; i.e., stage 0/I/ II colorectal cancer patients. Our study solely involved patients with stage $0(n=79)$, I $(n=80)$, or II $(n=123)$ colorectal cancer. Moreover, the established predictive model exhibited sensitivity of $>95 \%$, and its specificity was also $>90 \%$ (Table 3 ). These results suggest that the predictive model established in our study may be superior to other biomarker candidates reported in previous studies.

The established predictive model was composed of 8 metabolites; i.e., pyruvic acid, glycolic acid, tryptophan, palmitoleic acid, fumaric acid, ornithine, lysine, and 3-hydroxyisovaleric acid. The multicollinearity of the metabolites could not be confirmed because their variance inflation factor (VIF) values were small (Figure 1). Therefore, a variety of the factors associated with colorectal cancer without any lymph node or distant metastasis might have independently contributed to the alterations in the blood levels of each metabolite. For example, the colorectal cancer patients had higher plasma levels of pyruvic acid, which is an intermediate of glycolysis, than the healthy volunteers (Supplementary Table 2). The colorectal cancer patients also had higher plasma levels of lactic acid, which is the end product of glycolysis, than the healthy volunteers (Supplementary Table 2). A previous proteomic analysis of colorectal 
Table 2: AUC, sensitivity, and specificity values of the metabolites that exhibited Bonferroni-corrected significant differences

\begin{tabular}{|c|c|c|c|}
\hline & AUC & Sensitivity & Specificity \\
\hline Pyruvic acid-meto-TMS & 0.93551 & $87.9 \%$ & $90.4 \%$ \\
\hline Glycolic acid-2TMS & 0.90352 & $92.6 \%$ & $78.0 \%$ \\
\hline Lactic acid-2TMS(/SI) & 0.88963 & $82.6 \%$ & $82.8 \%$ \\
\hline Fumaric acid-2TMS(/SI) & 0.83445 & $74.1 \%$ & $80.8 \%$ \\
\hline Ornithine-4TMS(/SI) & 0.76751 & $68.8 \%$ & $76.3 \%$ \\
\hline Sucrose-8TMS & 0.7613 & $74.8 \%$ & $66.0 \%$ \\
\hline Fructose-meto-5TMS(2) & 0.71667 & $71.6 \%$ & $65.3 \%$ \\
\hline Arabinose-meto-4TMS & 0.71089 & $65.3 \%$ & $69.1 \%$ \\
\hline $\begin{array}{l}\text { 2-ketoglutaric acid-meto- } \\
\text { 2TMS }\end{array}$ & 0.67883 & $64.9 \%$ & $66.3 \%$ \\
\hline Sorbose-meto-5TMS(1) & 0.66413 & $67.7 \%$ & $61.9 \%$ \\
\hline Palmitoleic acid-TMS & 0.65756 & $55.3 \%$ & $69.4 \%$ \\
\hline Tryptophan-3TM(/SI) & 0.6573 & $45.0 \%$ & $81.1 \%$ \\
\hline Cysteine-3TMS & 0.64901 & $79.1 \%$ & $46.4 \%$ \\
\hline Xylose-meto-4TMS(2) & 0.6352 & $51.4 \%$ & $71.1 \%$ \\
\hline 2-aminobutyric acid-2TMS & 0.62659 & $72.7 \%$ & $50.5 \%$ \\
\hline Lysine-4TMS & 0.62194 & $39.0 \%$ & $81.8 \%$ \\
\hline Malic acid-3TMS(/SI) & 0.62077 & $42.2 \%$ & $77.0 \%$ \\
\hline Threitol-4TMS & 0.62045 & $39.0 \%$ & $80.4 \%$ \\
\hline Maltose-meto-8TMS(1) & 0.38827 & $12.4 \%$ & $96.2 \%$ \\
\hline Elaidic acid-TMS & 0.6098 & $53.9 \%$ & $67.0 \%$ \\
\hline Uric acid-4TMS & 0.60658 & $51.1 \%$ & $69.4 \%$ \\
\hline Isocitric acid-4TMS & 0.60463 & $35.5 \%$ & $85.6 \%$ \\
\hline meso-erythritol-4TMS & 0.60291 & $42.9 \%$ & $79.0 \%$ \\
\hline Valine-2TMS(/SI) & 0.595 & $47.2 \%$ & $69.4 \%$ \\
\hline Leucine-2TMS & 0.59334 & $49.3 \%$ & $65.6 \%$ \\
\hline $\begin{array}{l}\text { 3-hydroxyisovaleric acid- } \\
\text { 2TMS }\end{array}$ & 0.59258 & $38.3 \%$ & $78.7 \%$ \\
\hline Xylitol-5TMS & 0.5882 & $62.8 \%$ & $56.4 \%$ \\
\hline Arabitol-5TMS & 0.58759 & $57.1 \%$ & $60.8 \%$ \\
\hline Proline-2TMS & 0.58217 & $54.6 \%$ & $61.9 \%$ \\
\hline
\end{tabular}

A simple linear regression analysis of the metabolites that exhibited Bonferroni-corrected significant differences was performed, and the AUC, sensitivity, and specificity values of these metabolites are shown in Table 2. AUC: area under the curve; TMS: trimethylsilyl group; SI: stable isotope; '-TMS': the number of TMS molecules bound to each metabolite via derivatization; '/SI': the metabolites whose peak intensity values were normalized using the corresponding stable isotopes. 
cancer tissues obtained from stage III colorectal cancer patients revealed the upregulation of glycolysis [18]. On the other hand, Hirayama et al. found that colorectal cancer tissue contained lower levels of pyruvic acid than normal colon tissue, although the concentration of lactic acid was increased in the colorectal cancer tissue [19]. This phenomenon is known as 'the Warburg effect'. The colorectal cancer tissue examined in the study by Hirayama et al. was collected from colorectal cancer patients with stage I to IV disease. The blood level of pyruvic acid also reflects the nutritional state of the body regardless of the presence/absence of cancer. The plasma level of fumaric acid, which is an intermediate of the tricarboxylic acid (TCA) cycle (which occurs downstream of glycolysis), was also found to be increased in colorectal cancer patients compared with healthy volunteers, and moreover, the levels of 2-ketoglutaric acid, malic acid, and isocitric acid, which are intermediates of the TCA cycle, were significantly higher in the colorectal cancer patients
(Supplementary Table 2). Glycolysis and the TCA cycle are important metabolic pathways for providing energy to cells, and the abovementioned findings indicate that information about local colorectal cancer-based alterations in nutritional conditions should be included in predictive models for colorectal cancer.

Palmitoleic acid, which is a $\omega-7$ unsaturated fatty acid, is abundant in dietary oils, and it is also produced from palmitic acid by $\Delta 9$ desaturase in the body. In the present study, the colorectal cancer patients displayed significantly lower levels of palmitoleic acid than the healthy volunteers (Supplementary Table 2). The colorectal cancer patients exhibited lower blood levels of total cholesterol than the healthy volunteers, but their blood levels of triglycerides were higher than those of the colorectal cancer patients (Supplementary Table 1), suggesting that there is no relationship between stage 0/I/II colorectal cancer and lipid metabolism abnormalities. Palmitoleic acid enhances insulin

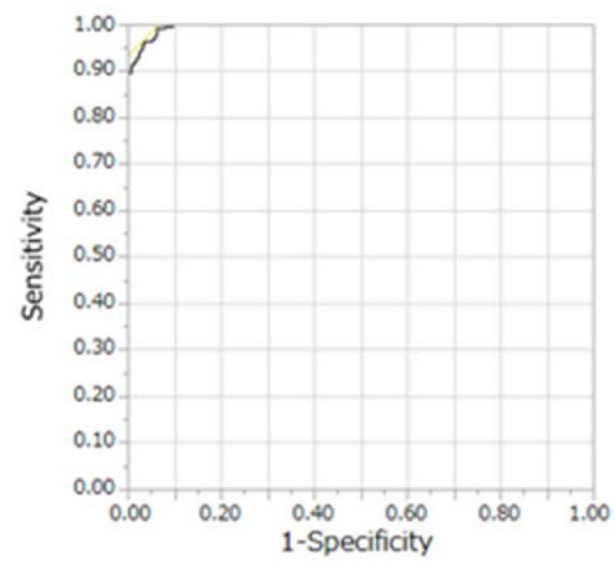

\begin{tabular}{ccccc}
\hline & Coefficient & S.E. & p-value & VIF \\
\hline Model & & & $<0.0001$ &
\end{tabular}

\begin{tabular}{lcccc}
$\quad$ (Intercept) & -8.99 & 2.32 & & \\
X1: Pyruvic acid-meto-TMS & 19.38 & 2.76 & $<0.0001$ & 1.63 \\
X2: Glycolic acid-2TMS & 82.33 & 13.20 & $<0.0001$ & 1.37 \\
X3: Tryptophan-3TM(/SI) & -6.86 & 1.80 & $<0.0001$ & 1.69 \\
X4: Palmitoleic acid-TMS & -67.48 & 18.79 & $<0.0001$ & 1.30 \\
X5: Fumaric acid-2TMS(/SI) & 296.32 & 63.38 & $<0.0001$ & 1.52 \\
X6: Ornithine-4TMS(/SI) & 9.69 & 2.12 & $<0.0001$ & 1.67 \\
X7: Lysine-4TMS & -5.76 & 1.63 & $<0.0001$ & 1.79 \\
X8: 3-hydroxyisovaleric acid-2TMS & -24.99 & 5.65 & 0.0030 & 1.16 \\
\hline
\end{tabular}

Figure 1: The ROC curve and data for the predictive model. The black line on the graph is the ROC curve for the predictive model. The AUC, sensitivity, and specificity values of the predictive model obtained via multiple logistic regression analysis were 0.996 , $99.3 \%$, and $93.8 \%$, respectively, and the optimal cut-off value was 0.19 . The coefficients, S.E., p-values, and VIF of this predictive model at the intercept and for each variable are shown in the table below the graph. S.E.: standard error; VIF: variance inflation factor; TMS: trimethylsilyl group; SI: stable isotope; '-TMS': the number of TMS molecules bound to each metabolite via derivatization; '/SI': the metabolites whose peak intensity values were normalized using the corresponding stable isotopes. 
Table 3: Sensitivity and specificity of the predictive model and tumor markers

\begin{tabular}{|c|c|c|c|c|c|}
\hline & & Stage $0 / \mathrm{I} / \mathrm{II}$ & Stage 0 & Stage I & Stage II \\
\hline \multirow[t]{3}{*}{ Sensitivity } & Model & $99.3 \%$ & $100 \%$ & $97.5 \%$ & $100 \%$ \\
\hline & CEA & $18.1 \%$ & $3.8 \%$ & $15.0 \%$ & $29.3 \%$ \\
\hline & CA19-9 & $9.3 \%$ & $6.4 \%$ & $6.3 \%$ & $13.1 \%$ \\
\hline \multirow[t]{3}{*}{ Specificity } & Model & $93.8 \%$ & $100 \%$ & $91.3 \%$ & $91.7 \%$ \\
\hline & CEA & $96.0 \%$ & $92.4 \%$ & $97.4 \%$ & $97 \%$ \\
\hline & CA19-9 & $95.6 \%$ & $95.5 \%$ & $97.4 \%$ & $94.4 \%$ \\
\hline
\end{tabular}

The sensitivity and specificity values of the predictive model obtained via multiple logistic regression analysis, and CEA and CA19-9, which were selected via simple linear regression analysis, are shown. Sensitivity and specificity were separately evaluated for stage 0-II (0/I/II), stage 0, stage I, and stage II disease. CEA: carcinoembryonic antigen; CA19-9: carbohydrate antigen 19-9.

sensitivity by suppressing inflammation and inhibiting the destruction of insulin-secreting pancreatic $\beta$ cells [20], and it also reduces obesity-related inflammation [21]. Thus, palmitoleic acid seems to exert antiinflammatory effects, and its administration to humans was shown to decrease blood CRP levels [22]. It has also been suggested that changes in a person's fatty acid profile might affect their risk of colorectal cancer. [23]. Therefore, a reduced level of palmitoleic acid could lead to altered inflammatory conditions in the body, and hence, influence the development of stage $0 / \mathrm{I} / \mathrm{II}$ colorectal cancer. Accordingly, it might be beneficial to include information about inflammatory conditions in predictive models for colorectal cancer.

In this study, a significant difference in smoking status was detected between the colorectal cancer patients and healthy volunteers, and the colorectal cancer patients had the high frequency of smoking status compared with healthy volunteers (Table 1). It has been reported that smoking habits affect the metabolite profile of the human body, including the blood [24-26]. In addition, habitual diet also seems to influence the serum metabolite profile [27]. Recently, a positive association between smoking and colorectal cancer was detected in Japanese [28], and so it might be necessary to include information about the lifestyle factors related to colorectal cancer without any lymph node or distant metastasis in predictive models for the disease. In a previous study, it was revealed that the power of a metabolite-based model to predict colorectal cancer was enhanced by incorporating four general clinical factors, age, gender, smoking status, and alcohol status, although the study in question included patients with stage I to IV colorectal cancer [29]. In addition, it was reported that the administration of aspirin and hormonal agents reduced the risk of colorectal cancer [30, 31]. Regarding aspirin, its use decreased the plasma level of oncometabolite 2-hydroxyglutarate in a randomized, double-blind, crossover trial [32], suggesting that medication might cause changes in the plasma levels of certain metabolites. In our study, information about the drugs being taken could not be collected for all subjects in the medication-positive group, so the positive/negative associations between particular medications and plasma metabolite levels should be discussed in the future.

In conclusion, a predictive model for detecting cases of colorectal cancer that do not involve lymph node or distant metastasis was established using a GC/QqQMS based-metabolomics approach. In this study, we focused on stage $0 / \mathrm{I} / \mathrm{II}$ colorectal cancer, and the diagnostic model was established. Accuracy of this diagnostic model for stage III/IV colorectal cancer has not been investigated, although the previous model [4] was evaluated for all stage (0/I/II/III/IV) colorectal cancer, so the established model may not be appropriate for stage III/IV colorectal cancer. The aim of this study is to establish the diagnostic model to discovery cases of colorectal cancer that do not involve lymph node or distant metastasis, and the metabolites on the model may be different between the previous and the current studies. Our predictive model exhibited high sensitivity and specificity in the present study population, and our results formed the basis of future's prospective studies for other cohorts, so its performance should be prospectively validated in other populations in which blood samples should be collected via the same procedure. In addition, it is also needed to perform the further validation study in general population based on healthy subjects and then to evaluate sensitivity of this model via endoscopy as the gold standard procedure. Specimens from colorectal cancer patients were collected from clinically-diagnosed subjects, so the results might not be representative of those in preclinical cancers involved in healthy population, even though the stages of cancers in the present study were 0 or I. Sensitivity calculated with clinically-diagnosed cancers generally tends to be overestimated than the true sensitivity for preclinical cancers in the population. Our study sample 
set had the significant differences in the plasma levels of total cholesterol, triglyceride, glucose, total bilirubin and AST between the colorectal cancer patients and healthy volunteers, so it is impossible to completely deny the involvement of these factors' alterations in the establishment of our model at this time. In the future, the contribution of blood biochemical factors including these factors to the diagnostic model should be also performed via the subset analysis in the validation study with the larger number of human samples. Finally, to enable improvements in QOL via the early detection of colorectal cancer, the practical utility of this predictive model should also be studied. Furthermore, the applicability of our model to screening examinations, for example, health checkups, should be examined.

\section{MATERIALS AND METHODS}

\section{Subjects}

This study was approved by the ethics committees at Kobe University Graduate School of Medicine and the National Cancer Center Japan. We included patients who were diagnosed with stage 0 , I or II colorectal cancer at the National Cancer Center Hospital; were histologically confirmed to have adenocarcinoma; and whose blood plasma was collected between May 2011 and July 2014 for research purpose. Patients who were simultaneously diagnosed with another type of cancer or had a history of cancer were excluded. The control plasma samples were obtained from healthy individuals who underwent cancer screening at the Research Center for Cancer Prevention and Screening, National Cancer Center, and whose blood plasma were available for researches. Blood was transferred into tubes containing EDTA-2Na as an anticoagulant. Blood collected from colorectal cancer patients was kept at room temperature for 15-30 min after gentle mixing, and then was kept at $4^{\circ} \mathrm{C}$. After $0.5-24 \mathrm{hr}$, the blood was centrifuged at $3,000 \mathrm{rpm}$ for $10 \mathrm{~min}$ at $4^{\circ} \mathrm{C}$, and plasma was obtained. Blood collected from healthy volunteers was kept at room temperature for about $30 \mathrm{~min}$ after gentle mixing, and then was kept at $4^{\circ} \mathrm{C}$. After $1-6 \mathrm{hr}$, the blood was centrifuged at $3,000 \mathrm{rpm}$ for $10 \mathrm{~min}$ at $4^{\circ} \mathrm{C}$, and plasma was obtained. Individuals who were diagnosed with cancer or had previously been diagnosed with cancer or colorectal polyps were excluded from the healthy control group. The colorectal cancer patients were classified into 5-year age groups $(40-44,45-49,50-54, \ldots, 80-84,85+)$, and healthy volunteers were selected by matching them as closely as possible with the colorectal cancer patients based on gender, age group, and the year of blood collection. All plasma samples were prospectively collected from individuals who had provided written informed consent to allow their blood samples to be used for research purposes and were stored at $-80^{\circ} \mathrm{C}$ at the National Cancer Center Biobank (the samples from the colorectal cancer patients) or the Research Center for Cancer Prevention and Screening (the samples from the healthy volunteers). We retrospectively selected 282 colorectal cancer patients and 291 healthy volunteers for our analyses based on the patient/healthy control selection criteria and collected their plasma samples. The subjects' characteristics are summarized in Table 1. Clinical staging was performed based on the Union for International Cancer Control TNM Classification (7th edition). The classification of histology and tumor location was conducted based on the Japanese Classification of Colorectal Carcinoma (8th edition). In this study, each sample was numbered in a blinded manner before metabolite extraction, and steps until peak identification and alignment were performed by using this blinded number.

\section{Chemicals and reagents}

${ }^{13} \mathrm{C}_{3}$-lactic acid, ${ }^{13} \mathrm{C}_{2}$-oxalic acid, ${ }^{2} \mathrm{H}_{3}$-sacrosine, ${ }^{2} \mathrm{H}_{8}$-valine, ${ }^{13} \mathrm{C}_{3}$-dihydroxyacetone, ${ }^{2} \mathrm{H}_{10}$-isoleucine, ${ }^{13} \mathrm{C}_{4}$-fumaric acid, ${ }^{13} \mathrm{C}_{4}$-malic acid, ${ }^{2} \mathrm{H}_{3}$-aspartic acid, ${ }^{13} \mathrm{C}_{5}$-glutamic acid, ${ }^{13} \mathrm{C}_{6}$-4-hydroxybenzoic acid, ${ }^{2} \mathrm{H}_{3}-$ lauric acid, ${ }^{13} \mathrm{C}_{5}$-ribose, ${ }^{13} \mathrm{C}_{2}$-taurine, ${ }^{2} \mathrm{H}_{4}$-citric acid, ${ }^{2} \mathrm{H}_{7}$ ornithine, ${ }^{13} \mathrm{C}_{6}$-tyrosine, ${ }^{13} \mathrm{C}_{6}$-dopa, ${ }^{2} \mathrm{H}_{6}$-kynurenine, ${ }^{2} \mathrm{H}_{8}-$ cystamine, and ${ }^{13} \mathrm{C}_{11}$-tryptophan were purchased from Cambridge Isotope Laboratories, Inc. (MA, USA). ${ }^{2} \mathrm{H}_{3}-$ 2-hydorxybutyric acid and 2-isopropylmalic acid were purchased from CDN isotopes (Quebec, CA) and Sigma Aldrich (Tokyo, Japan), respectively. The compounds were dissolved in methanol, as shown in Supplementary Table 3 , and then the obtained solution was used as an extraction solution. A standard alkane series mixture (C7 to $\mathrm{C} 33$ ) and octafluoronaphthalene (OFN) were purchased from Restek Co. (PA, USA) and Shimadzu Co. (Kyoto, Japan), respectively. Methoxyamine hydrochloride and N-methyl-N-trimethylsilyl-trifluoroacetamide (MSTFA), which were used for the derivatization, were obtained from Sigma-Aldrich and GL Science (Tokyo, Japan), respectively.

\section{Sample preparation}

To extract low molecular weight metabolites, 50 $\mu \mathrm{L}$ of plasma were mixed with $270 \mu \mathrm{L}$ of the extraction solution (Supplementary Table 3), and then the mixture was shaken at $1,200 \mathrm{rpm}$ for $30 \mathrm{~min}$ at $37^{\circ} \mathrm{C}$, before being centrifuged at $2,000 \mathrm{x}$ g for $10 \mathrm{~min}$ at $4^{\circ} \mathrm{C}$. One hundred $\mu \mathrm{L}$ of the obtained supernatant were transferred to a clean tube and then dried for $3 \mathrm{hr}$ with a centrifugal evaporator. For oximation, $80 \mu \mathrm{L}$ of $20 \mathrm{mg} /$ $\mathrm{ml}$ methoxyamine hydrochloride dissolved in pyridine were added to the tube and then sonicated for $10 \mathrm{~min}$, before being shaken at $1,200 \mathrm{rpm}$ for $90 \mathrm{~min}$ at $30^{\circ} \mathrm{C}$. The mixture was centrifuged at $2,000 \mathrm{x}$ g for $10 \mathrm{~min}$ at $20^{\circ} \mathrm{C}$, and then $40 \mu \mathrm{L}$ of the resultant supernatant were subjected to GC/QqQMS, as described in the next section. 


\section{GC/QqQMS procedure}

The GC/QqQMS analysis was performed on an AOC-6000 (Shimadzu Co.) and a GCMS-TQ8040 (Shimadzu Co.) equipped with a BPX-5 capillary column (internal diameter: $30 \mathrm{~m} \times 0.25 \mathrm{~mm}$; film thickness: 0.25 $\mu \mathrm{m}$; SEG, Victoria, Australia). In the AOC-6000, $20 \mu \mathrm{L}$ of MSTFA were added to the sample supernatant, and then the mixture was incubated at $750 \mathrm{rpm}$ for $30 \mathrm{~min}$ at $37^{\circ} \mathrm{C}$, before $1.0 \mu \mathrm{L}$ of the derivatized solution was injected into the GCMS-TQ8040. During the GCMSTQ8040 analysis, the inlet temperature was kept at $250^{\circ} \mathrm{C}$, and helium was used as a carrier gas at a constant flow rate of $39.0 \mathrm{~cm}$ per sec. The injector split ratio was set to $1: 30$. The GC column temperature was programmed to remain at $60^{\circ} \mathrm{C}$ for $2 \mathrm{~min}$ and then rise from $60^{\circ} \mathrm{C}$ to $330^{\circ} \mathrm{C}$ at a rate of $15^{\circ} \mathrm{C}$ per $1 \mathrm{~min}$, before being kept at $330^{\circ} \mathrm{C}$ for $3 \mathrm{~min}$. The total $\mathrm{GC}$ run time was $23 \mathrm{~min}$. The transfer line and ion-source temperature were $280^{\circ} \mathrm{C}$ and $200^{\circ} \mathrm{C}$, respectively. The ionization voltage was $70 \mathrm{eV}$. Argon gas was used as a collision-induced dissociation gas. The MRM cycle time was set at 5 cycles/sec to allow accurate peak area assessment. The metabolite detection was performed using the Smart Metabolites Database (Shimadzu, Co.), which contained the relevant MRM method file and data regarding the GC analytical conditions, MRM parameters, and retention index employed for the metabolite measurement. To correct the retention time, the Automatic Adjustment of Retention Time (AART) function of the GCMSsolution software (Shimadzu Co.) and a standard alkane series mixture (C7 to $\mathrm{C} 33$ ) were used. The peak identification was performed automatically and then confirmed manually based on the specific precursor and product ions, and the retention time. The database used in this study includes data about 215 peaks from 153 metabolites, 22 corresponding stable isotopes, and 2-isopropylmalic acid. Due to derivatizationinduced variations, 35 metabolites produce 2 peaks each, and 2 metabolites have 3 peaks each. The peak intensity of each metabolite was normalized to that of the internal standard; i.e., 4-hydroxybenzoic acid- ${ }^{13} \mathrm{C}_{6}-2 \mathrm{TMS}$. Regarding the metabolites that we identified based on the stable isotopes, their peak intensities were corrected using the corresponding stable isotopes. In this study, 22 stable isotopes were used, but some native metabolites were not detected in the subjects' plasma. During the normalization process, 22 metabolites were corrected using both the internal standard and the corresponding stable isotopes. Then, the data that exhibited the best co-efficient of variation $(\mathrm{CV})$ during the quality control (QC) process was adopted for the study. Based on the elimination process conducted as part of the $\mathrm{QC}$ protocol described in the next section, 64 metabolites were finally selected as reliable targets for statistical evaluation. The MRM parameters for the detected metabolites were shown in Supplementary Table 4. The 2-isopropylmalic acid contained in the extraction solution was also used to evaluate the stability of our GC/QqQMS analysis system.

\section{Quality controls}

In order to ensure the reproducibility of the acquired metabolomic data, a $\mathrm{QC}$ procedure is required [33]. We established a specific data cleansing and QC protocol before the analyses. Commercially available pooled plasma (Kohjin-Bio Co., Saitama, Japan) was used as the QC sample. All QC plasma samples were derived from the same product lot. One QC sample was analyzed with each batch of 10 study samples. The QC samples were prepared and analyzed in the same manner as the study samples. An OFN sample (concentration: $100 \mathrm{pg} / \mu \mathrm{L}$ ) was also analyzed in each batch of 10 study samples. The criteria used to eliminate samples and metabolites from the study were as follows: 1) samples for which the area ratios of the internal standard; i.e., 4-hydroxybenzoic acid- ${ }^{13} \mathrm{C}_{6}-2 \mathrm{TMS}$, were $<0.5$ or $>1.5$ times the median value for the batch; 2) samples in which $\geq 2$ stable isotopes that were subjected to metabolite extraction could not be identified; 3) metabolites that could not be detected in $\geq 1$ QC or study samples; 4) metabolites for which the CV for all QC samples was $>30 \%$ after normalization using the internal standard, or $>20 \%$ after normalization with the corresponding stable isotopes; and 5) batches in which a signal value of $<2,000$ is detected in $100 \mathrm{pg} / \mu \mathrm{L}$ of OFN.

\section{Statistical analysis}

Numerical data regarding age, BMI, and the blood test results are presented as the mean and standard deviation (S.D.) for each group, and were compared using the Student's t-test. Categorical data concerning sex, cancer stage, or medical questionnaire responses are presented as distribution charts, and were compared using Pearson's chi-square test. In the comprehensive analysis of metabolites, the plasma levels of each metabolite are presented as mean and S.D. values and were also compared using the Wilcoxon's rank sum test. Ratios of the level of a particular metabolite in the colorectal cancer patients to that seen in the healthy volunteers are shown as fold change values. For clinical variables, $p$-values of $<0.05$ were considered to indicate a significant difference. For the metabolite analysis, p-values indicating significant differences were adjusted using the Bonferroni method. The stepwise method was used to select variables for the multivariate analysis. The multicollinearity of the selected variables was assessed by calculating VIF values. The multivariate analysis was performed using multiple logistic regression analysis. Receiver operating characteristic (ROC) curve analysis was used to evaluate the diagnostic performance of the resultant regression model based on its AUC, sensitivity, and specificity 
values. These analyses were performed using the default conditions of JMP12 (SAS Institute Inc.).

\section{ACKNOWLEDGMENTS}

We are deeply grateful to Reiko Tanaka (Shimadzu Corporation, Kyoto Japan) for her work as an experimental assistant. We also thank the patients and healthy volunteers, who kindly donated their blood for research purposes, and the National Cancer Center Biobank for providing the samples. The National Cancer Center Biobank is supported by the National Cancer Center Research and Development Fund, Japan.

\section{CONFLICTS OF INTEREST}

The authors declare that no conflicts of interest associated with this manuscript exist.

\section{GRANT SUPPORT}

This study was supported in part by a Grant-inAid for Scientific Research (B) from the Japan Society for the Promotion of Science (JSPS) [M.Y.]; a Grant-inAid for Scientific Research (C) from the JSPS [S.N.]; and the Practical Research for Innovative Cancer Control from the Japan Agency for Medical Research and Development (AMED) [S.N. and M.Y.]; the Medical Research and Development Programs Focused on Technology Transfer: Development of Advanced Measurement and Analysis Systems (SENTAN) from AMED [S.N., T.K., Y.U., T.S., K.O., Y.Y., K.S., N.T.O., N.O., and M.Y.]; and the AMED-CREST from AMED [S.N., T.K., T.A., and M.Y.].

\section{REFERENCES}

1. World Cancer Report 2014. International Agency for Research on Cancer/World Health Organization. Edited by Stewart BW and Wild CP.

2. Matsuda T, Marugame T, Kamo K, Katanoda K, Ajiki W, Sobue T, Japan Cancer Surveillance Research Group. Cancer incidence and incidence rates in Japan in 2004: based on data from 14 population based cancer registries in the Monitoring of Cancer Incidence in Japan (MCIJ) project. Japanese Journal of Clinical Oncology. 2010; 40: 1192-1200.

3. Nambiar PR, Gupta RR, Misra V. An "Omics" based survey of human colon cancer. Mutation Research, 2010; 693: 3-18.

4. Nishiumi S, Kobayashi T, Ikeda A, Yoshie T, Kibi M, Izumi Y, Okuno T, Hayashi N, Kawano S, Takenawa T, Azuma T, Yoshida M. A novel serum metabolomics-based diagnostic approach for colorectal cancer. PLoS ONE. 2012; 7: e40459.
5. Yoshida M, Hatano N, Nishiumi S, Irino Y, Izumi Y, Takenawa T, Azuma T. Diagnosis of gastroenterological diseases by metabolome analysis using gas chromatographymass spectrometry. Journal of Gastroenterology 2012; 47 : 9-20.

6. Rochfort, S. Metabolomics reviewed: A new "Omics" platform technology for systems biology and implications for natural products research. Journal of Natural Products. 2005; 68: 1813-1820.

7. Suzuki M., Nishiumi S., Matsubara A., Azuma T., Yoshida M. Metabolome analysis for discovering biomarkers of gastroenterological cancer. J Chromatogr B Analyt Technol Biomed Life Sci. 2014; 966: 59-69.

8. Nishiumi S, Suzuki M, Kobayashi T, Matsubara A, Azuma T, Yoshida M. Metabolomics for Biomarker Discovery in Gastroenterological Cancer. Metabolites. 2014; 4: 547-571.

9. Tsugawa H, Tsujimoto Y, Sugitate K, Sakui N, Nishiumi $\mathrm{S}$, Bamba T, Fukusaki E. Highly sensitive and selective analysis of widely targeted metabolomics using gas chromatography/triple-quadrupole mass spectrometry. Journal of Bioscience and Bioengineering. 2014; 117: 122-128.

10. Tsugawa $H$, Bamba $T$, Shinohara $M$, Nishiumi $S$, Yoshida M, Fukusaki E. Practical Non-targeted Gas Chromatography/Mass Spectrometry-based Metabolomics Platform for Metabolic Phenotype Analysis. Journal of Bioscience and Bioengineering. 2011; 112: 292-298.

11. Quéro A, Jousse C, Lequart-Pillon $\mathrm{M}$, Gontier E, Guillot X, Courtois B, Courtois J, Pau-Roblot C. Improved stability of TMS derivatives for the robust quantification of plant polar metabolites by gas chromatography-mass spectrometry. J Chromatogr B Analyt Technol Biomed Life Sci. 2014; 970: 36-43.

12. Noctor G, Bergot G. Mauve C. Thominet D. LelargeTrouverie C. Prioul JL. A comparative study of amino acid measurement in leaf extracts by gas chromatographytime of flight-mass spectrometry and high performance liquid chromatography with fluorescence detection. Metabolomics. 2007; 3: 161-174.

13. Ritchie SA, Ahiahonu PW, Jayasinghe D, Heath D, Liu J, Lu Y, Jin W, Kavianpour A, Yamazaki Y, Khan AM, Hossain M, Su-Myat KK, Wood PL, et al. Reduced levels of hydroxylated, polyunsaturated ultra long-chain fatty acids in the serum of colorectal cancer patients: implications for early screening and detection. BMC Medicine. 2010; 8: 13.

14. Miyagi Y, Higashiyama M, Gochi A, Akaike M, Ishikawa T, Miura T, Saruki N, Bando E, Kimura H, Imamura F, Moriyama M, Ikeda I, Chiba A, et al. Plasma free amino acid profiling of five types of cancer patients and its application for early detection. PLoS ONE. 2011; 6: e24143.

15. Li F, Qin X, Chen H, Qiu L, Guo Y, Liu H, Chen G, Song G, Wang X, Li F, Guo S, Wang B, Li Z. Lipid profiling for early diagnosis and progression of colorectal cancer using direct-infusion electrospray ionization Fourier transform 
ion cyclotron resonance mass spectrometry. Rapid Communications in Mass Spectrometry. 2013; 27: 24-34.

16. Lin Y, Ma C, Liu C, Wang Z, Yang J, Liu X, Shen Z, $\mathrm{Wu}$ R. NMR-based fecal metabolomics fingerprinting as predictors of earlier diagnosis in patients with colorectal cancer. Oncotarget. 2016; 7: 29454-29464. doi: 10.18632/ oncotarget.8762.

17. Uchiyama K, Yagi N, Mizushima K, Higashimura Y, Hirai Y, Okayama T, Yoshida N, Katada K, Kamada K, Handa O, Ishikawa T, Takagi T, Konishi H, et al. Serum metabolomics analysis for early detection of colorectal cancer. Journal of Gastroenterology. 2016.

18. Bi X, Lin Q, Foo TW, Joshi S, You T, Shen HM, Ong CN, Cheah PY, Eu KW, Hew CL. Proteomic analysis of colorectal cancer reveals alterations in metabolic pathways: mechanism of tumorigenesis. Molecular \& Cellular Proteomics. 2006; 5: 1119-1130.

19. Hirayama A, Kami K, Sugimoto M, Sugawara M, Toki N, Onozuka H, Kinoshita T, Saito N, Ochiai A, Tomita M, Esumi H, Soga T. Quantitative metabolome profiling of colon and stomach cancer microenvironment by capillary electrophoresis time-of-flight mass spectrometry. Cancer Research. 2009; 69: 4918-4925.

20. Yang ZH, Miyahara H, Hatanaka A. Chronic administration of palmitoleic acid reduces insulin resistance and hepatic lipid accumulation in KK-Ay Mice with genetic type 2 diabetes. Lipids in Health and Disease. 2011; 10: 120.

21. Chan KL, Pillon NJ, Sivaloganathan DM, Costford SR, Liu Z, Théret M, Chazaud B, Klip A. Palmitoleate Reverses High Fat-induced Proinflammatory Macrophage Polarization via AMP-activated Protein Kinase (AMPK). Journal of Biological Chemistry. 2015; 290: 16979-16988.

22. Bernstein AM, Roizen MF, Martinez L. Purified palmitoleic acid for the reduction of high-sensitivity $\mathrm{C}$-reactive protein and serum lipids: a double-blinded, randomized, placebo controlled study. Journal of Clinical Lipidology. 2014; 8: 612-617.

23. Theodoratou E, McNeill G, Cetnarskyj R, Farrington SM, Tenesa A, Barnetson R, Porteous M, Dunlop M, Campbell H. Dietary fatty acids and colorectal cancer: a case-control study. American Journal of Epidemiology. 2007; 166: 181-195.

24. Gu F, Derkach A, Freedman ND, Landi MT, Albanes D, Weinstein SJ, Mondul AM, Matthews CE, Guertin KA, Xiao Q, Zheng W, Shu XO, Sampson JN, et al. Cigarette smoking behaviour and blood metabolomics. International Journal of Epidemiology. 2016; 45:1421-1432.
25. Hsu PC, Lan RS, Brasky TM, Marian C, Cheema AK, Ressom HW, Loffredo CA, Pickworth WB, Shields PG. Metabolomic profiles of current cigarette smokers. Molecular Carcinogenesis. 2017; 56:594-606.

26. Chen Q, Deeb RS, Ma Y, Staudt MR, Crystal RG, Gross SS. Serum Metabolite Biomarkers Discriminate Healthy Smokers from COPD Smokers. PLoS ONE. 2015; 10: e0143937.

27. Playdon MC, Sampson JN, Cross AJ, Sinha R, Guertin KA, Moy KA, Rothman N, Irwin ML, Mayne ST, StolzenbergSolomon R, Moore SC. Comparing metabolite profiles of habitual diet in serum and urine. The American Journal of Clinical Nutrition. 2016; 104: 776-789.

28. Mizoue T, Inoue M, Tanaka K, Tsuji I, Wakai K, Nagata C, Tsugane S; Research Group for the Development, Evaluation of Cancer Prevention Strategies in Japan. Tobacco smoking and colorectal cancer risk: an evaluation based on a systematic review of epidemiologic evidence among the Japanese population. Japanese Journal of Clinical Oncology. 2006; 36: 25-39.

29. Zhu J, Djukovic D, Deng L, Gu H, Himmati F, Chiorean EG, Raftery D. Colorectal cancer detection using targeted serum metabolic profiling. Journal of Proteome Research. 2014;13: 4120-4130.

30. Drew DA, Cao Y, Chan AT. Aspirin and colorectal cancer: the promise of precision chemoprevention. Nature Reviews Cancer. 2016; 16: 173-186.

31. Johnson JR, Lacey JV Jr, Lazovich D, Geller MA, Schairer C, Schatzkin A, Flood A. Menopausal hormone therapy and risk of colorectal cancer. Cancer Epidemiology, Biomarkers \& Prevention. 2009; 18: 196-203.

32. Liesenfeld DB, Botma A, Habermann N, Toth R, Weigel C, Popanda O, Klika KD, Potter JD, Lampe JW, Ulrich CM. Aspirin Reduces Plasma Concentrations of the Oncometabolite 2-Hydroxyglutarate: Results of a Randomized, Double-Blind, Crossover Trial. Cancer Epidemiology, Biomarkers \& Prevention. 2016; 25: 180-187.

33. Dunn WB, Broadhurst D, Begley P, Zelena E, FrancisMcIntyre S, Anderson N, Brown M, Knowles JD, Halsall A, Haselden JN, Nicholls AW, Wilson ID, Kell DB, Goodacre $\mathrm{R}$ et al. Procedures for large-scale metabolic profiling of serum and plasma using gas chromatography and liquid chromatography coupled to mass spectrometry. Nature Protocols. 2011; 6: 1060-1083. 